\title{
ORIGINAL
}

\section{MORTALIDAD YCOSTES ASOCIADOS A LA DEMORA DEL TRATAMIENTO QUIRÚRGICO POR FRACTURA DE CADERA}

\author{
Iñigo Etxebarria-Foronda (1), Javier Mar (2), Arantzazu Arrospide (2) y Jaime Ruiz \\ de Eguino (3).
}

(1) Cirugía Ortopédica y Traumatología. Hospital Alto Deba.

(2) Unidad de Investigación. Hospital Alto Deba.

(3) Dirección económico-financiera. Hospital Alto Deba.

\begin{abstract}
RESUMEN
Fundamentos: La intervención quirúrgica en las fracturas de cadera suele demorarse varios días. Nuestro trabajo tiene dos objetivos. Estudiar la estancia preoperatoria de los pacientes ingresados por fractura de cadera en los hospitales vascos durante el año 2010 y medir su posible asociación con la mortalidad intrahospitalaria, y estimar el coste económico que supone la estancia preoperatoria.

Métodos. Se realizó un estudio observacional analizando los siguientes datos del Conjunto Mínimo Básico de Datos (CMBD): hospital, edad, sexo, estancia preoperatoria, estado al alta, diagnósticos asociados y procedimiento quirúrgico. Además, se estudió el índice de comorbilidad de Charlson y se calculó el coste unitario de la estancia pre y postoperatoria mediante modelos de regresión múltiple.

Resultados. Se analizó una muestra de 1.856 intervenciones. La estancia preoperatoria fue de 2,7 días y la postoperatoria de 9,7 días. El coste medio por ingreso fue de $12.552,12$ euros. El coste medio de la estancia preoperatoria fue de 1295,5 euros. La mortalidad fue del $5 \%$.

Conclusiones. La estancia preoperatoria no es un factor estadísticamente asociado con la mortalidad, aunque aumenta significativamente el coste total. No encontramos una asociación entre la demora quirúrgica y la mortalidad, aunque sí tiene una clara influencia en el coste total del proceso. La reducción del tiempo prequirúrgico permite ahorrar costes.

Palabras clave: Fractura de cadera. Coste. Mortalidad. Procedimientos quirúrgicos ortopédicos
\end{abstract}

\author{
Correspondencia \\ Iñigo Etxebarria \\ Servicio de Cirugía Ortopédica y Traumatología \\ Hospital Alto Deba \\ Avda. Navarra 16 \\ 20500. Mondragón. Gipuzkoa. \\ ietxe@yahoo.es
}

\section{ABSTRACT}

\section{Cost and Mortality associated to the Surgical Delay of Patients with a Hip Fracture. Spain}

Background: Hip fractures surgery is often delayed for several days The present work has two objectives. The first one is to determine the preoperative hospital length of stay of patients with a hip fracture in our region and its possible correlation with an increase in mortality rate Secondly we assessed the healthcare expenditure associated to this preoperative period.

Methods: We carried out an observational, retrospective study in which all hip fractures attended in the Basque Country throughout 2010 were assessed by the Minimun Basic Data Set (MSBD): hospital, age, sex, preoperative hospital length, status at hospital discharge, comorbidities and surgical procedure. Furthermore we studied the Charlson Index and the total cost associated stratified by pre and post-operative period using a multiple model regression.

Results: A total of 1856 surgical procedures were analyzed. Average pre and post-operative length of stay was 2.7 and 9.7 days respectively. The mean total cost per hospital admission was $12,552 €$, with $1,295.5 €$ corresponding to the preoperative period. The duration of preoperative stay is not associated with a significant reduction in mortality, although it did with an increase in the total cost.

Conclusions: Preoperative hospital stay in patients with hip fracture is still challenging. We could not find an association between the delays in surgical procedures and hospital mortality, although it seems to be associated with an increased the total procedure cost. These results can serve as a foundation for setting up strategies to decrease the length of hospital stay, not only for cost-saving purposes.

Key words: Hip Fracture. Cost. Mortality. Orthopedics surgical procedures. 


\section{INTRODUCCIÓN}

Las fracturas de cadera son lesiones que tienen gran trascendencia en salud pública por su incidencia, su impacto en la mortalidad y su repercusión socioeconómica. Su principal factor de riesgo es la enfermedad osteoporótica $^{1}$, cuya prevalencia aumenta de forma exponencial con el envejecimiento de la población. Además, en los ancianos son mucho más frecuentes las caídas que actúan como factor precipitante. Se estima que hasta un tercio de los sujetos mayores de 64 años refieren haber tenido una caída el último año, que se eleva a la mitad en los pacientes mayores de 80 y que incluso un $25 \%$ de estos tienen más de una caída ${ }^{2}$. La incidencia total de las fracturas en una población aumenta de forma paralela al aumento de su esperanza de vida ${ }^{3}$. Sin embargo, hay trabajos que recogen una reducción o estabilización de las mismas en los últimos años, cuando se ajustan los resultados por la edad ${ }^{4}$, postulándose como posible factor causal el aumento en el consumo de fármacos para la osteoporosis ${ }^{5}$.

La fractura de cadera es una situación clínica grave que implica una importante mortalidad y pérdida de la calidad de vida ${ }^{6}$. Prácticamente la totalidad de los pacientes requiere una solución quirúrgica que en muchas ocasiones sufre una demora de varios días, lo que se ha relacionado con un aumento de las complicaciones y de la mortalidad $^{7,8}$. La tasa de mortalidad de estos pacientes duplica la de las personas de la misma edad sin fractura, con los problemas respiratorios y cardiovasculares como principales causas de defunción dentro de los primeros 30 días ${ }^{7}$. Hay trabajos que recogen que la mitad de estas muertes son causadas por la situación clínica del paciente en el momento de producirse la fractura, pero el resto se deben a complicaciones postoperatorias que se podrían evitar ${ }^{8}$. En este debate, se plantea si la demora quirúrgica es un determinante de la mortalidad que puede ser mejorado con estrategias que aceleren el proceso asistencial. Además, hay que tener en cuenta que la demora incrementa el coste total del episodio.

El primer objetivo del estudio es analizar la estancia preoperatoria de los pacientes ingresados por las fracturas de cadera en los hospitales vascos durante el ejercicio 2010 y medir su asociación con la mortalidad intrahospitalaria. El segundo es medir el coste económico que supone la duración de la estancia preoperatoria.

\section{MATERIAL Y MÉTODOS}

Diseño observacional analizando las fracturas de cadera atendidas por el Servicio Vasco de Salud durante el año 2010. Se excluyeó a los pacientes que no fueron intervenidos durante el ingreso o los que fueron dados de alta al ser trasladados a otro hospital de agudos. Se estudiaron las siguientes variables del Conjunto Mínimo Básico de Datos (CMBD): hospital del ingreso, edad, sexo, la estancia total, la estancia preoperatoria, el estado al alta, los diagnósticos y el procedimiento quirúrgico.

Dado que el objetivo del estudio fue analizar la asociación de la estancia preoperatoria con la mortalidad se utilizó la medida de la comorbilidad para identificar causas de muerte ajenas a la cirugía. La comorbilidad describe el efecto de todas las otras enfermedades que un paciente individual pudiera tener en la mortalidad hospitalaria. De los diferentes índices descritos se decidió utilizar el índice de comorbilidad de Charlson por ser el más utilizado en nuestro medio ${ }^{9}$. Este índice predice la mortalidad de un paciente en función de la presencia de enfermedades crónicas asociadas tales como las enfermedades del corazón, el sida o el cáncer hasta un total de 22 condiciones ${ }^{10}$. En el artículo original de Charlson se incluye un ajuste en función de la edad para los seguimientos prolongados que consiste en añadir un punto al índice por cada década posterior a los 50 años. En nuestro caso 
no lo utilizamos para evitar que prácticamente todos los pacientes tuviesen un índice superior a 2 , que indica la presencia de comorbilidad y el índice perdiese capacidad discriminativa.

A partir de los datos de la contabilidad analítica de cada uno de los once hospitales públicos del País Vasco se calculó el coste unitario de la estancia pre-operatoria y de la estancia postoperatoria. Para ello se desagregó el coste total de la hospitalización del servicio de traumatología en costes de la estancia y coste de la intervención. El coste por hora de quirófano del servicio de traumatología en cada hospital se calculó dividiendo los costes totales entre las horas ocupadas en 2010. El coste de cada procedimiento se obtuvo sumando el coste del tiempo de quirófano más el coste de los dispositivos. Para conseguir el primero, se tuvieron en cuenta el coste total del quirófano, las horas totales de quirófano y el tiempo medio utilizado con cada procedimiento en traumatología. Los tiempos medios fueron de 4,4 horas para la reducción cerrada con fijación interna; 4,8 horas para la reducción abierta con fijación interna; 5,2 horas para la prótesis total y 4,8 para la prótesis parcial. El coste de la estancia preoperatoria consistió directamente en el coste de la estancia de traumatología en cada hospital sin los costes del procedimiento quirúrgico. El coste total postoperatorio de cada paciente se obtuvo sumando el coste de la estancia y el coste del procedimiento. De esta manera se evitó imputar a la estancia preoperatoria el coste de la intervención ya que la disminución de la demora quirúrgica no modifica el coste del procedimiento.

En el análisis descriptivo se clasificó a los pacientes por grupos de edad y los hospitales como generales o comarcales. Además, los pacientes fueron divididos en función del índice de Charlson en dos grupos: los que tenían puntuación 0 y aquellos con puntuación o superior a 0 . Se utilizaron frecuencias absolutas y relativas para la des- cripción de las variables cualitativas. Las variables continuas fueron descritas mediante su media y la desviación estándar de la media. A continuación se realizó un análisis univariante en el que se analizó qué factores influyeron de manera significativa en la mortalidad hospitalaria en este tipo de intervenciones. En el caso de las variables cualitativas se compararon las tasas de fallecimiento para cada categoría de las variables mediante el estadístico exacto de Fisher ( 2 categorías) o el estadístico $\mathrm{chi}^{2}$ (más de 2 categorías). En el análisis se incluyeron el tipo de hospital, el sexo, ser mayor de 75 años, tener un índice de Charlson mayor que 0 y el tipo del primer diagnóstico y procedimiento detallados en el CMBD. Además, para las variables continuas, como las estancias pre y postoperatorias y el coste total del ingreso, se compararon los valores medios medianos según el estado al alta (vivos o fallecidos al alta) mediante la prueba no paramétrica de la suma de rangos de Wilcoxon por ser variables con sesgo positivo.

Basándonos en los resultados previos, los factores con valor estadísticamente significativo en el análisis univariante fueron incluidos en una regresión logística con el fallecimiento durante el ingreso como variable dependiente. Las variables claves, como el sexo o la duración de la estancia preoperatoria, también fueron incluidas como factores predictores de la mortalidad con el fin de determinar su asociación con la mortalidad intrahospitalaria. Finalmente, con el objetivo de conocer el peso de las características estudiadas en el cómputo final del coste total del ingreso se realizó un análisis multivariante mediante una regresión lineal. El coste total fue la variable dependiente y se incluyeron como factores predictores todas las variables categóricas analizadas además de la estancia preoperatoria. Todos los análisis estadísticos fueron realizados con el paquete estadístico SPSS versión 19. 


\section{RESULTADOS}

Durante el año 2010, en el conjunto de los centros del Servicio Vasco de Salud, hubo 2.108 ingresos por fracturas de cadera. El $11,95 \%$ fueron excluidos del estudio por no incluir intervención durante el ingreso o bien por tener un traslado a otro hospital de agudos como motivo del alta. Finalmente se analizó una muestra de 1.856 sujetos. En la tabla 1 se muestran sus características. El $75,3 \%$ de los pacientes fueron mujeres y la edad media fue de 81,7 (DE 11,7) años. Según el índice de Charlson el 67,5\% de los sujetos intervenidos no tenía ningún tipo de comorbilidad. La estancia preoperatoria de estos pacientes fue de 2,7 (DE 3,3) días y la postoperatoria de 9,7 (DE 6,1) días. E1 $82,4 \%$ de los pacientes fue intervenido en un hospital general. La estancia preoperatoria en hospitales comarcales fue de 2,13 días (DE 2,0) y en los hospitales generales de 2,79 días (DE 3,54). La diferencia fue estadísticamente significativa $(\mathrm{p}<0.001)$.

En la tabla 2 se describen los costes desagregados de los 1.856 pacientes. El coste total incurrido para los 1.856 pacientes fue de 23.306.089,0 $€$ (tabla 2), dando una media de $12.557,2 €(\mathrm{DE}=7.160,7 €)$

Tabla 1

Características de la muestra

\begin{tabular}{|c|c|c|}
\hline & $\mathrm{n}$ & $\%$ \\
\hline Hospital comarcal & 327 & 17,6 \\
\hline Hospital general & 1529 & 82,4 \\
\hline Hombre & 459 & 24,7 \\
\hline Mujer & 1397 & 75,3 \\
\hline Menores de 70 años & 194 & 10,5 \\
\hline Entre 70 y 74 años & 85 & 4,6 \\
\hline Entre 75 y 79 años & 272 & 14,7 \\
\hline Entre 80 y 84 años & 436 & 23,5 \\
\hline Entre 85 y 89 años & 486 & 26,2 \\
\hline Mayores de 90 años & 383 & 20,6 \\
\hline Destino a domicilio habitual & 1326 & 71,4 \\
\hline Hospitalización a domicilio & 39 & 2,1 \\
\hline Traslado a un hospital de media y larga estancia & 323 & 17,4 \\
\hline Residencia social & 44 & 2,4 \\
\hline Fallecido & 93 & 5,0 \\
\hline Alta voluntaria & 1 &, 1 \\
\hline Otras causas & 30 & 1,6 \\
\hline Índice de Charlson & 1253 & 67,5 \\
\hline$>0$ & 603 & 32,5 \\
\hline \multirow{2}{*}{\begin{tabular}{|rr} 
Primer diagnóstico & Fractura intracapsular \\
Fractura pertrocanterea o intertrocanterea
\end{tabular}} & 906 & 48,81 \\
\hline & 802 & 43,21 \\
\hline Fractura subtrocanterea & 148 & 7,97 \\
\hline \multicolumn{3}{|l|}{ Primer procedimiento } \\
\hline Reducción cerrada de fractura con fijación interna, fémur & 691 & 37,23 \\
\hline Reducción abierta de fractura con fijación interna, fémur & 393 & 21,17 \\
\hline $\begin{array}{l}\text { Sustitución parcial de cadera } \\
\end{array}$ & 131 & 7,06 \\
\hline Revisión de sustitución de cadera & 569 & 30,66 \\
\hline Otro procedimiento & 72 & 3,88 \\
\hline Total & 1.856 & 100 \\
\hline
\end{tabular}


Tabla 2

Componentes de costes de los ingresos por las fracturas de cadera

\begin{tabular}{|l|r|r|r|}
\hline & Pre & Post & Total \\
\hline Estancia media & 2,7 & 9,7 & 12,4 \\
\hline Coste Unitario & 479,8 & $1.161,0$ & $1.012,7$ \\
\hline Coste total/paciente & $1.295,5$ & $11.261,7$ & $12.557,2$ \\
\hline Número de altas & 1.856 & 1.856 & 1.856 \\
\hline Coste total & $2.404 .373,8$ & $20.901 .715,2$ & $23.306 .089,0$ \\
\hline Coste total estancias & & & $11.042 .309,1$ \\
\hline Coste total cirugía & & & $12.263 .779,8$ \\
\hline Coste cirugía/paciente & & & $6.607,6$ \\
\hline
\end{tabular}

por ingreso. El coste de la estancia preoperatoria fue de 2.404.373,8 $€$ con un coste medio por ingreso de $1.295,5 €$ (DE $1.535,4 €)$ y de $479,8 €$ por día de estancia. Cuando se incluyó el coste de la cirugía, el coste por día de estancia total subió a $1.012,7 €$. El coste de la cirugía fue de $6.607,6 €$ por paciente. La distribución de frecuencias del coste total por paciente siguió una distribución lognormal con una forma asimétrica y una cola mayor en los valores superiores (figura 1).
En el análisis univariante realizado resultaron variables asociadas a la mortalidad la edad y el índice de Charlson, además del tipo de procedimiento realizado (tabla 3). La diferencia en la estancia media postoperatoria fue estadísticamente significativa, no así la diferencia en la estancia media preoperatoria.

En las tablas 4 y 5 se muestran los resultados de las regresiones logística y lineal respectivamente. En la regresión logística se observa que el hecho de ser mujer reduce significativamente la probabilidad de muerte casi a la mitad $(\mathrm{OR}=0,61)$. Además, los sujetos mayores de 75 años en comparación a los menores de 75 tienen casi 4 veces más posibilidad de fallecer durante el ingreso $(\mathrm{OR}=3,5)$ y un índice de Charlson de 1 o más supone 2 veces más probabilidad de muerte que los de índice $0(\mathrm{OR}=2,2)$. Las diferencias en la estancia preoperatoria no fueron un factor estadísticamente significativo.

Figura 1

Frecuencias del coste total por paciente según grupo de coste

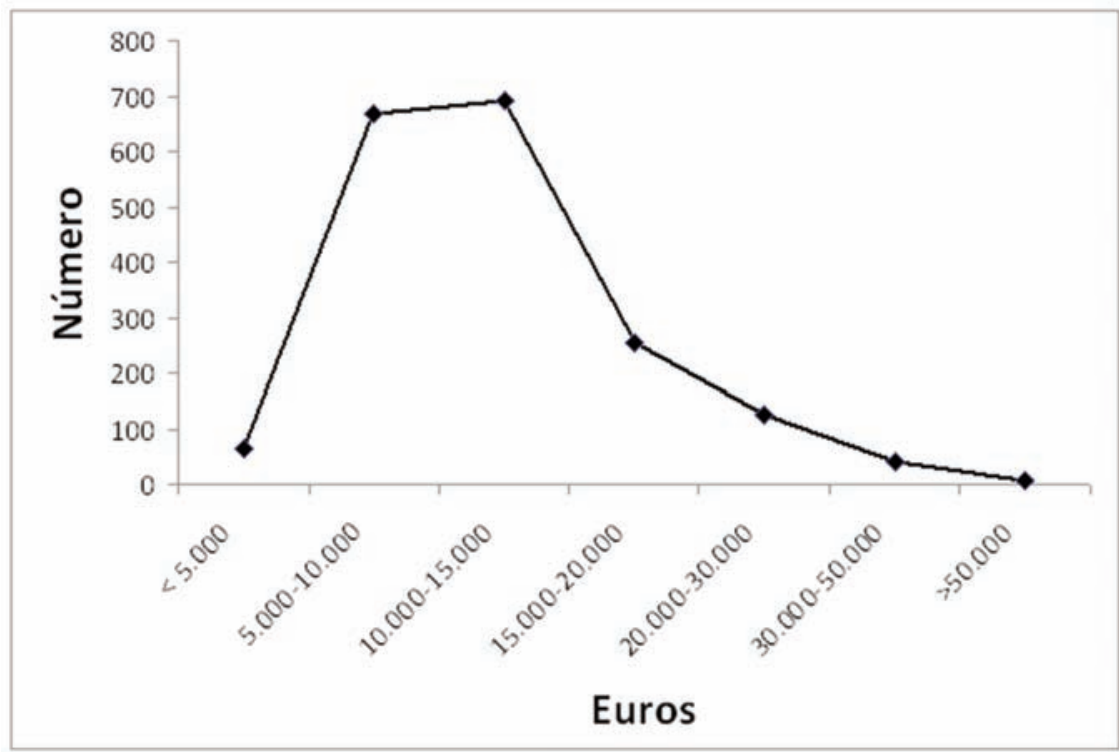


Tabla 3

Análisis univariante con respecto a la mortalidad durante el ingreso

\begin{tabular}{|c|c|c|c|c|c|c|}
\hline & & \multicolumn{2}{|c|}{$\begin{array}{c}\text { Fallecido } \\
(\mathrm{n}=93)\end{array}$} & \multicolumn{2}{|c|}{$\begin{array}{l}\text { No fallecido } \\
\quad(n=1763)\end{array}$} & \multirow{2}{*}{\begin{tabular}{|c|c}
$\mathrm{N}$ \\
1.856 \\
$\mathrm{p}$
\end{tabular}} \\
\hline & & $\mathrm{n}$ & $\%$ & $\mathrm{n}$ & $\%$ & \\
\hline \multirow{2}{*}{ Hospital } & Hospital comarcal & 16 & 4,9 & 311 & 95,1 & \\
\hline & Hospital general & 77 & 5,0 & 1452 & 95,0 & 1,000 \\
\hline \multirow{2}{*}{ Sexo } & Hombre & 31 & 6,8 & 428 & 93,2 & \\
\hline & Mujer & 62 & 4,4 & 1335 & 95,6 & 0,063 \\
\hline \multirow{2}{*}{ Edad } & Menores de 75 años & 5 & 1,8 & 274 & 98,2 & \\
\hline & De 75 años o más & 88 & 5,6 & 1489 & 94,4 & 0,005 \\
\hline \multirow{2}{*}{ Índice de Charlson } & 0 & 45 & 3,6 & 1208 & 96,4 & \\
\hline & $>0$ & 48 & 8,0 & 555 & 92,0 & 0,000 \\
\hline \multirow{3}{*}{ Primer diagnóstico } & Fractura intracapsular & 53 & 5,8 & 853 & 94,2 & \\
\hline & Fractura pertrocanterea o intertrocanterea & 35 & 4,4 & 767 & 95,6 & \\
\hline & Fractura subtrocanterea & 5 & 3,4 & 143 & 96,6 & 0,238 \\
\hline \multirow{6}{*}{ Primer procedimiento } & Reducción cerrada de fractura con fijación interna, fémur & 21 & 3,0 & 670 & 97,0 & \\
\hline & Reducción abierta de fractura con fijación interna, fémur & 14 & 3,6 & 379 & 96,4 & \\
\hline & Sustitución parcial de cadera & 8 & 6,1 & 123 & 93,9 & \\
\hline & Revisión de sustitución de cadera & 35 & 6,2 & 534 & 93,8 & \\
\hline & Otro procedimiento & 15 & 20,8 & 57 & 79,2 & 0,000 \\
\hline & & Media & $\mathrm{DE}$ & Media & $\mathrm{DE}$ & \\
\hline \multicolumn{2}{|l|}{ Estancia preoperatoria } & 3,20 & 4,87 & 2,65 & 3,23 & \\
\hline \multicolumn{2}{|l|}{ Estancia postoperatoria } & 9,23 & 11,66 & 9,77 & 5,68 & \\
\hline \multirow{2}{*}{\multicolumn{2}{|c|}{ Coste total }} & $12.408,7$ & $10.882,3$ & $12.559,7$ & $6.913,6$ & \\
\hline & & Mediana & RI & Mediana & RI & $\mathrm{p}$ \\
\hline \multicolumn{2}{|l|}{ Estancia preoperatoria } & 2 & 4 & 2 & 3 & 0,909 \\
\hline \multicolumn{2}{|l|}{ Estancia postoperatoria } & 6 & 10 & 8 & 5 & 0,000 \\
\hline \multicolumn{2}{|l|}{ Coste total } & $10.440,0$ & $11.554,7$ & $11.308,0$ & $5.692,0$ & 0,024 \\
\hline
\end{tabular}

RI: Rango intercuartílico 
Tabla 4

Regresión logística para la probabilidad de muerte durante el ingreso

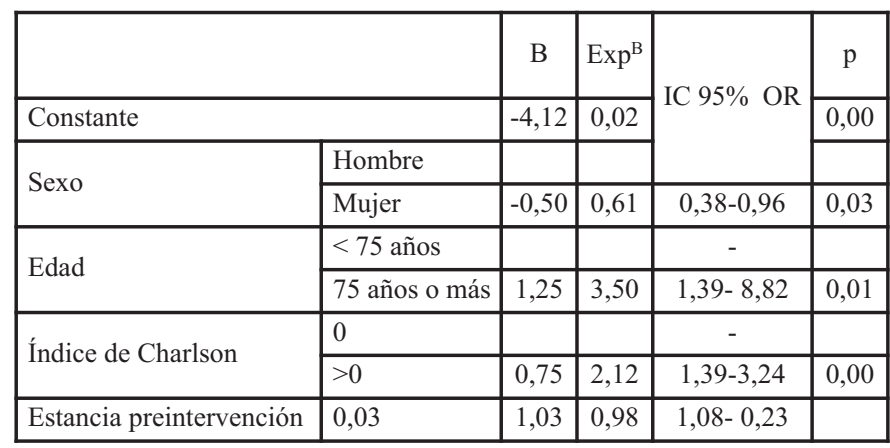

Tabla 5

Regresión lineal para predecir el coste total del ingreso

\begin{tabular}{|c|c|c|c|c|}
\hline & & B & $\begin{array}{c}\text { IC } 95 \% \\
\text { para Exp }{ }^{\text {B }}\end{array}$ & $\mathrm{p}$ \\
\hline Constante & & $10.013,437$ & $8.680,330-11.346,544$ & 0,000 \\
\hline Sexpo & Hombre & & & \\
\hline $\operatorname{sex} 0$ & Mujer & $-794,996$ & $-1.482,339--107,654$ & 0,023 \\
\hline & $<75$ años & & - & \\
\hline Luad & 75 años o más & $1.178,172$ & $351,325-2.005,019$ & 0,005 \\
\hline Índice de Charleon & 0 & & - & \\
\hline & $>0$ & 971,715 & $351,600-1.591,830$ & 0,002 \\
\hline Estancia preintervención & 0,03 & 977,841 & $891,251-1.064,432$ & 0,000 \\
\hline
\end{tabular}

En cuanto al coste del ingreso, la regresión lineal indica que tanto la edad como el sexo, el índice de Charlson o la estancia preoperatoria aumentan significativamente el coste total. Por la reducción de cada día de estancia total se ahorrarían de media $977,84 €$ si se ajustan los datos estadísticamente por las otras variables. Esta cifra no ajustada fue de $1.012,7 €$, tal como se observa en la tabla 2.

\section{DISCUSIÓN}

El principal hallazgo de este estudio es la falta de asociación entre la demora quirúrgica y la mortalidad hospitalaria de los pacientes intervenidos por fractura de cadera. La demora debe ser la mínima siempre que permita llevar a cabo la intervención en condiciones adecuadas, aunque la literatura muestra resultados controvertidos al medir su asociación con la mortalidad. Mientras dos estudios canadienses y dos norteamericanos no aprecian una relación estadísticamente significativa ${ }^{11-14}$, otros trabajos señalan que la mayor demora en la cirugía produce un aumento de la mortalidad ${ }^{15-17}$. Aunque la mayor parte de los trabajos de la literatura consisten, como el nuestro, en estudios transversales basados en el CMBD, es difícil comparar los resultados, ya que las metodologías no son siempre las mismas sin que se pueda encontrar un patrón definido. Algunos estudios miden la mortalidad durante el ingreso hospitalario mientras que 
otros continúan el seguimiento hasta los 30 días, los seis meses o el año. La variable demora se ha procesado en algunos estudios como variable continua y en otros se ha hecho categórica. Como señalan algunos autores, es difícil sacar conclusiones de la heterogénea información existente ${ }^{7,18}$.

En nuestra serie, los pacientes esperaron una media de 2,7 días para ser intervenidos, lo cual está en concordancia con otros estudios realizados en nuestro país ${ }^{19}$. No es fácil valorar si esa demora es adecuada o no. En principio, la falta de significación estadística descrita entre la diferencia en la estancia previa a la cirugía entre fallecidos y no fallecidos podría interpretarse en el sentido de que no ha determinado cambios en la mortalidad. Hay estudios que consideran que una cirugía precoz es aquella que se realiza dentro de las primeras 24 horas $^{20-}$ 22 , otros dentro de las primeras $48^{13,23}$, y algunos dentro de las 72 horas tras el ingre$\mathrm{so}^{24}$. Incluso hay autores que señalan que realizar la intervención dentro de las 6 primeras horas mejora los resultados de mortalidad y funcionales ${ }^{25}$. La mayoría de los trabajos reconocen que una cirugía "temprana" podría tener como punto de corte las primeras 48 horas e influye de una manera positiva en las tasas de mortali$\operatorname{dad}^{15,20,22,25-32}$. Sin embargo, también hay autores que ponen en duda tal aseveración ${ }^{14,23,33,34}$. Existe controversia por tanto acerca de si el retraso en realizar la cirugía en este tipo de fracturas genera más mortalidad, pero lo que sí parece claro es que una cirugía temprana está asociada a un menor número de complicaciones, mejores resultados funcionales, menor estancia hospitalaria y menos reingresos, lo cual indudablemente está asociado a un menor coste $^{14,35}$. La mayoría de las guías de práctica clínica recomiendan que la intervención se realice dentro de las primeras 24-48 horas $^{36-37}$, por lo que podemos considerar los tiempos de demora de nuestra muestra como superiores a lo deseable.
No es fácil analizar el motivo de la diferencia estadísticamente significativa de la estancia preoperatoria entre los hospitales comarcales y los generales. Por un lado los pacientes con problemas de comorbilidad son derivados a los generales. Por otro, es posible que la gestión en hospitales más pequeños sea más ágil a la hora de movilizar los recursos necesarios para llevar a cabo la intervención quirúrgica.

El impacto económico de la reducción de la estancia preoperatoria es importante ya que el coste de cada día es de 477,8 euros. La no inclusión del coste de la intervención determina una reducción desde los 1.012,7 € que es el coste de cada día de la estancia total del paciente. A pesar de ese ajuste contable su dimensión es relevante y debe de ser tenida en cuenta. Sin embargo, su impacto económico se debe valorar en función del coste de las posibles alternativas organizativas que permitiesen disminuirla. Para lo cual es necesario el análisis de la demora quirúrgica que se centra en dos causas. Por un lado están los condicionantes clínicos que recomienden retrasar el acto quirúrgico para mejorar la preparación del paciente. El otro motivo de retraso son los problemas organizativos y de limitaciones de personal de los que depende la disponibilidad de quirófanos. En un reciente trabajo se aprecia que el principal motivo es la ausencia de quirófano disponible, muy por encima de las razones médicas que pudieran contraindicar la cirugía precoz ${ }^{19}$. También hay comunicaciones en sentido inverso, en las que se muestra que la principal causa de demora es fundamentalmente clínica ${ }^{38}$.

Desde el punto de vista clínico, la comorbilidad de los pacientes aumenta el riesgo de complicaciones y de mortalidad y muchos autores recomiendan su previa estabilización antes de proceder al acto quirúrgi$\mathrm{co}^{12,19,21,39,40}$. En nuestra serie llama la atención que la mayoría de los pacientes $(67,5 \%)$ no tenían ningún tipo de comorbilidad, lo cual, posiblemente, tiene relación con la falta de registro de la comorbilidad en sus infor- 
mes de alta. Es difícil señalar otra explicación a este hallazgo en una muestra de pacientes con una media de 82 años. En España el CMBD presenta limitaciones importantes como fuente de datos $\operatorname{crí}^{\prime} \cos ^{41}$. Por otro lado, nuestros resultados muestran que los pacientes con mayor índice de comorblidad tienen mayor probabilidad de muerte. Por tanto, desde esta perspectiva clínica, las estrategias de mejora de la estancia preoperatoria deben ir encaminadas al manejo adecuado de estos problemas médicos añadidos, con la interacción de todo el personal implicado en el proceso, para que, garantizando la seguridad del paciente, se eviten demoras innecesarias que perjudiquen su curso clínico.

Respecto a los condicionantes estructurales u organizativos, está claro que la aparición de las fracturas de cadera es imprevisible y que, por tanto, su atención quirúrgica no se puede programar como la cirugía electiva. El hecho de planificar de una manera reglada la solución quirúrgica puede conllevar un aumento del coste del proceso por aumento de personal o de disponibilidad de quirófanos, pero es importante tener en cuenta que si se consigue reducir la demora también se van a reducir los costes.

A modo de conclusión podríamos decir que nuestra estancia preoperatoria en pacientes con fractura de cadera es mejorable. Que pese a ello, no encontramos una relación entre la demora quirúrgica y la mortalidad de nuestros pacientes. No obstante, sí que hay una clara influencia en el coste total del proceso. En este sentido, son necesarias estrategias que reduzcan la estancia previa no sólo para ahorrar costes, sino también para mejorar la recuperación funcional de los pacientes.

\section{BIBLIOGRAFÍA}

1. Melton LJ 3rd, Thamer M, Ray NF, Chan JK, Chesnut CH 3rd, Einhorn TA, Johnson CC, Raisz LG, Silverman SL, Siris ES. Fractures attributable to osteoporosis: report from the National Osteoporosis Foundation. J Bone Miner Res. 1997; 12: 16-23.
2. Duaso E, Casas A, Formiga F, Lázaro del Nogal M, Salvà A, Marcellán T, Navarro C. Unidades de prevención de caídas y de fracturas osteoporóticas. Propuesta del Grupo de Osteoporosis, Caídas y Fracturas de la Sociedad Española de Geriatría y Gerontología. Rev Esp Geriatr Gerontol. 2011; 46:268-274.

3. Cooper C, Campion G, Melton LJ 3rd. Hip fractures in the elderly: a world wide projection. Osteoporosis Int. 1992; 2:285-289.

4. Cooper C, Cole ZA, Holroyd CR, Earl SC, Harvey NC, Dennison EM, Melton LJ, Cummings SR, Kanis JA. Secular trends in the incidence of hip and other osteoporotic fractures. Osteoporos Int. 2011; 22: 127788 .

5. Etxebarria-Foronda I, Mar J, Arrospide A, EsnalBaza E. Trends in the incidence of hip fractures in women in the Basque country. Arch Osteoporos. 2010; 5: $131-137$

6. Johnell O, Kanis JA. An estimate of the worldwide prevalence and disability associated with osteoporotic fractures. Osteoporos Int. 2006; 17: 1726-33.

7. González-Montalvo JL, Alarcón T, Hormigo-Sánchez AI. Why do hip fracture patients die? Med Clin (Barc). 2011; 137: 355-60.

8. Foss NB, Kehlet H. Mortality analysis in hip fracture patients: implications for design of future outcome trials. Br J Anaesth. 2005; 94: 24-9.

9. Alvarez-Nebreda ML, Jiménez AB, Rodríguez P, Serra JA. Epidemiology of hip fracture in the elderly in Spain. Bone. 2008 Feb;42(2):278-85.

10. Charlson ME, Pompei P, Ales KL, Mackenzie CR. A new method of classifying prognostic comorbidity in longitudinal studies: Development and validation. J Chron Dis. 1987; 40: 373-83.

11. Majumdar SR, Beaupre LA, Johnston DWC, Dick DA, Cinats JG, Jiang HX. Lack of association between mortality and timing of surgical fixation in elderly patients with hip fracture. Med Care. 2006;44:552-9.

12. Bergeron E, Lavoie A, Moore L, Bamvita JM, Ratte $\mathrm{S}$, Gravel $\mathrm{C}$, et al. Is the delay to surgery for isolated hip fracture predictive of outcome in efficient systems? J Trauma. 2006;60:753-7.

13. Hoenig H, Rubenstein LV, Sloane R, Horner R, Kahn K. What is the role of timing in the surgical and rehabilitative care of community-dwelling older persons with acute hip fracture? Arch Intern Med. 1997; 157:513-20 
14. Orosz GM, Magaziner J, Hannan EL, Morrison RS, Koval K, Gilbert M, et al. Association of timing of surgery for hip fracture and patient outcomes. JAMA. 2004;291:1738-43.

15. Novack V, Jotkowitz A, Etzion O, Porath A. Does delay in surgery after hip fracture lead to worse outcomes? A multicenter survey. Int J Qual Health Care. 2007; 19:170-6.

16. Maggi S, Siviero P, Wetle T, Besdine RW, Saugo M, Crepaldi G. A multicenter survey on profile of care for hip fracture: predictors of mortality and disability. Osteoporos Int. 2010;21:223-31.

17. Shiga T, Wajima Z, Ohe Y. Is operative delay associated with increased mortality of hip fractured patients? Systematic review, meta-analysis, and metaregression. Can J Anesth. 2008;55:146-54.

18. Bernal-Delgado E, Librero J, Peiró S, Leutscher E, Ridao M, Martínez N. Reparación de la fractura de cadera en personas mayores. Asociación entre el tiempo hasta la intervención y la mortalidad en la fractura de cadera en el Sistema Nacional de Salud. Documento de trabajo 03-2009. Disponible en www.atlasvpm.org.

19. Vidán MT, Sánchez E, Gracia Y, Marañón E, Vaquero J, Serra JA. Causes and effects of surgical delay in patients with hip fracture. A cohort study. Ann Intern Med. 2011; 155: 226-33.

20. Rogers FB, Shackford SR, Keller MS. Early fixation reduces morbidity and mortality in elderly patients with hip fractures from low-impact falls. J Trauma. 1995; 39: 261-5.

21. Kenzora JE, McCarthy RE, Lowell JD, Sledge CB. Hip fracture mortality: relation to age, treatment, preoperative illness, time of surgery and complications. Clin Orthop. 1984; 186: 45-56.

22. Perez JV, Warwick DJ, Case CP, Bannister GC. Death after proximal femoral fracture: an autopsy study. Injury. 1995; 26: 237-40.

23. Parker MJ, Pryor GA. The timing of surgery for proximal femoral fractures. J Bone Joint Surg [Br]. 1992; 74B: 203-5.

24. Kitamura S, Hasegawa Y, Suzuki S, Sasaki R, Iwata H, Wingstrand H, Thorngren KG. Functional outcome after hip fracture in Japan. Clin Orthop. 1998; 348: 29-36.

25. Dorotka R, Schoechtner H, Buchinger W. The influence of immediate surgical treatment of proximal femoral fractures on mortality and quality of life. Ope- ration within six hours of the fracture versus later than six hours. J Bone Joint Surg [Br]. 2003; 85: 1107-13.

26. Weller I, Wai EK, Jaglal S, Kreder HJ. The effect of hospital type and surgical delay on mortality after surgery for hip fracture. J Bone Joint Surg [Br]. 2005; 87: 361-6.

27. Sund R, Liski A. Quality effects of operative delay on mortality in hip fracture treatment. Qual Saf Health Care. 2005; 14: 371-377.

28. Lefaivre KA, Macadam SA, Davidson DJ, Gandhi R, Chan H, Broekhuyse HM. Length of stay, mortality, morbidity and delay to surgery in hip fractures. J Bone Joint Surg [Br]. 2009; 91: 922-7.

29. Zuckerman JD, Skovron ML, Koval KJ, Aharonoff G, Frankel VH. Postoperative complications and mortality associated with operative delay in older patients who have a fracture of the hip. J Bone Joint Surg Am. 1995; 77: 1551-6.

30. McGuire KJ, Berstein J, Polsky D, Silber JH. The 2004 Marshall Urist award: delays until surgery after hip fracture increases mortality. Clin Orthop Relat Res. 2004; 428: 294-301

31. Simunovic N, Devereaux PJ, Sprague S, Guyatt GH, Schemitsch E, Debeer J, Bhandari M. Effect of early surgery after hip fracture on mortality and complications: systematic review and meta-analysis. CMAJ. 2010; 182: 1609-16.

32. Sánchez-Crespo MR, Bolloque R, Pascual-carra A, Pérez-Aguilar MD, Rubio-Lorenzo M, Alonso-Aguirre MA, Sánchez-Juan P. Mortalidad al año en fracturas de cadera y demora quirúrgica. Rev Esp Cir Ortop Traumatol. $2010 ; 54: 34-38$

33. Rae HC, Harris IA, McEvoy L, Todorova T. Delay to surgery and mortality after hip fracture. ANZ J Surg. 2007; 77: 889-91.

34. Rodriguez-Fernández P, Adarraga-Cansino D, Carpintero P. Effects of delayed hip fracture surgery on mortality and morbidity in elderly patients. Clin Orthop Relat Res. 2011; 469: 3218-3221.

35. Al-Ani AN, Samuelsson B, Tidermark J, Norling A, Ekström W, Cederholm T, Hedström M. Early operation on patients with a hip fracture improved the ability to return to independient living. A prospective study of 850 patients. J Bone Joint Surg Am. 2008; 90: 1436-42.

36.Scottish Intercollegiate Guidelines Network. "Management of hip fracture in older people. A National Clinical Guideline". Scotland 2009. Disponible en URL: http://www.sign.ac.uk/pdf/sign111.pdf. 
37. The British Orthopaedic Association. The care of patients with fragility fracture. London, 2007. Disponible en http://www.boa.ac.uk/site/show/publications

38. Charalambous CP, , Yarwood S, Paschalides C, Siddique I, Hirst P, Paul A. Factors delaying surgical treatment of hip fractures in the elderly patients. Ann R Coll Surg Engl. 2003; 85: 117-9.

39. Hamlet WP, Lieberman JR, Freedman EL, Dorey FJ, Fletcher A, Johnson EE. Influence of health status and the timing of surgery on mortality in hip fracture patients. Am J Orthop. 1997; 26: 621-627.

40. Stoddart J, Horne G, Devane P. Influence of preoperative medical status and delay to surgery on death following hip fracture. ANZ J Surg. 2002; 72: 405-407.

41. Rodríguez-Rieiro C, Rodríguez Pérez P, Granado de la Orden S, Moreno Moreno M, García AC, Sánchez-Gómez A. In-hospital mortality rates after CABG by autonomous regions in Spain. Int $\mathrm{J}$ Health Care Qual Assur. 2011;24(4):300-7. 